\title{
Polarization Characteristics of Compact SOI Rib Waveguide Racetrack Resonators
}

\author{
Isa Kiyat, Student Member, IEEE, Atilla Aydinli, Member, IEEE, and Nadir Dagli, Senior Member, IEEE
}

\begin{abstract}
We report on the development of compact optical racetrack resonators on silicon-on-insulator (SOI) rib waveguides. We make use of large-cross-section waveguides instead of photonic wire waveguides. We fabricated resonators with bending radii down to $20 \mu \mathrm{m}$ and characterized for both transverse-electric and transverse-magnetic polarizations. Different polarization characteristics were analyzed and related to the modal shape of the SOI waveguide. These compact resonators show large free spectral ranges $(3.0 \mathrm{~nm})$, high finesse $(19)$, and $Q$-factor $(28000)$ values.
\end{abstract}

Index Terms-Integrated optics, optical wavelength filter, resonator, silicon-on-insulator (SOI) technology.

\section{INTRODUCTION}

C OMPACT low-loss optical racetrack resonators using high-index contrast waveguides are promising candidates for wavelength-selective applications. Ring resonators in different material systems such as AlGaAs-GaAs [1], GaInGaP-InP [2], and submicron-thick silicon-on-insulator (SOI) [3]-[5] have been realized for a variety of wavelength-selective devices and high-speed modulators [6]. Due to its compatibility with mature silicon based microelectronics technology and its high-index contrast, SOI is an ideal platform for compact devices that may be used in large scale integration of optical circuits. Among the high-index contrast material platforms, SOI provides a low-cost alternative to molecular beam epitaxy or metal-organic chemical vapor deposition grown III-V alloy systems.

Most devices employing ring resonators have been fabricated on submicron-thick SOI with wire waveguides due to large index difference in this material system [3], [4]. Such devices, while compact, require sophisticated and expensive submicron lithography to define the waveguides as well as nanometric coupling gaps. Furthermore, the submicron cross-sectional area of the waveguides makes it difficult to couple light into and out of waveguides increasing insertion loss, dramatically. Finally, propagation loss in wire waveguides scale with the cube of the index perturbation due to surface roughness on nanometric waveguides [7]. On the other hand, waveguides with larger cross sections are more tolerant to fabrication problems, and are easier to fabricate at lower cost. Recently, a polarization-independent large radius $(400 \mu \mathrm{m})$ ring using a large cross section was studied [8] resulting in limited free spectral range (FSR)

Manuscript received March 21, 2005; revised June 28, 2005.

I. Kiyat and A. Aydinli are with Turk Telekom Bilkent Laboratories, Department of Physics, Bilkent University, Ankara 06800, Turkey (e-mail: kiyat@fen.bilkent.edu.tr).

N. Dagli is with Department of Electrical and Computer Engineering, University of California, Santa Barbara, CA 93106 USA.

Digital Object Identifier 10.1109/LPT.2005.856410 of $0.19 \mathrm{~nm}$. In a recent paper, we also realized resonators based on the same technology [9]. These were high- $Q$ resonators (up to 119000) suitable for a certain type of application such as quantum optics. Obtaining such high- $Q$ values requires very low radiation loss which resulted in large circumference leading to FSRs not larger than $0.63 \mathrm{~nm}$. Furthermore, by definition, the bandwidth of such resonators is very narrow. In many telecom applications, devices with larger FSRs and and wider bandwidths are required. The current limited bandwidth and FSR of SOI rib waveguide resonators can be overcome by implementing compact devices with smaller radii.

In this work, we report on a systematic study of compact large cross section SOI racetrack resonators. These resonators were realized using a waveguide structure with strong light confinement. A set of resonators with varying radii were designed, fabricated, and characterized for large FSR values. Particular attention was paid to polarization characteristics of the devices. Resonators with radii as small as $20 \mu \mathrm{m}$, with FSRs as large as $3.0 \mathrm{~nm}$ have been realized.

\section{REALIZATION OF SOI RACETRACK RESONATORS}

In this study, commercially available ultrauniform $\mathrm{Si}$ on $\mathrm{SiO}_{2}$ layers have been used [9]. We determined the dimensions of single-mode SOI waveguides through extensive numerical calculations using the beam propagation method (BPM) employing a commercially available software package. The waveguide dimensions and calculated optical intensity profiles of both polarizations are shown in the insets of Fig. 1. An important point in the design of racetrack resonators is the estimation of bending loss. We calculated the bending loss using a two-dimensional (2-D) analysis based on Marcuse's equation for bending slab waveguides. In this approach, the three-dimensional waveguide structure is reduced to 2-D using the effective index method as explained in [10], and the resulting analysis leads to bending loss of $-10 \log \left(\exp \left(-\alpha_{\text {bend }} \Delta \theta R\right)\right)$ in decibels for a given angular section $\Delta \theta$ (in radians) as a function of bending radius. The optical bending loss coefficient $\alpha_{\text {bend }}$ and details of the analysis are given in [9] and [10]. The bending losses obtained for both transverse-electric (TE) and transverse-magnetic (TM) polarizations are displayed in Fig. 1. The results show that the bending losses drastically decrease for radii larger than 30 and $80 \mu \mathrm{m}$ for TM and TE modes, respectively. The relatively small bending loss for TM when compared to TE is due to stronger lateral confinement of the TM mode, as can be seen by comparing the mode profiles in the inset of Fig. 1.

The design of the racetrack resonator can be finalized, once the geometry of the waveguides to be used as well as an estimation of the optical loss and the choice of the coupling gap and 


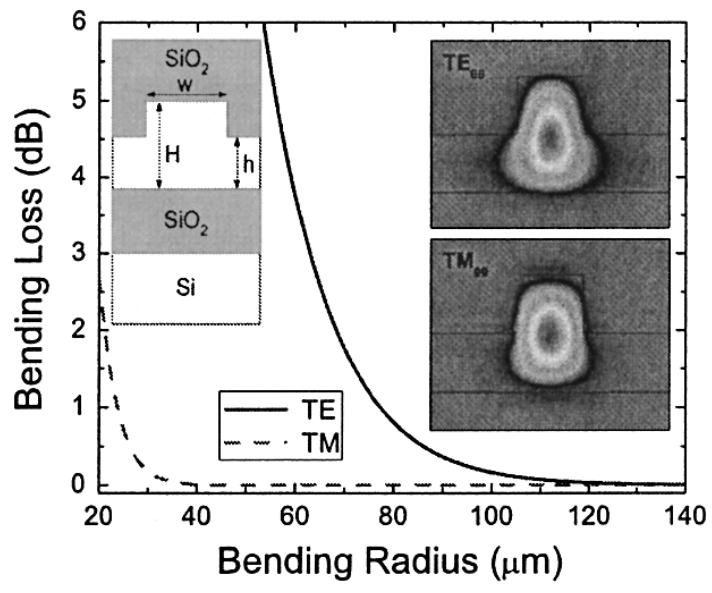

Fig. 1. Analytically calculated bending loss for a $90^{\circ}$ bend as a function of bending radius. The insets show critical waveguide dimensions ( $w$ and $H$ are $1.0 \mu \mathrm{m}$ and $h$ is $0.5 \mu \mathrm{m})$ and calculated TE and TM mode profiles for the SOI waveguide. The effective refractive indexes are calculated to be 3.3546 and 3.3402 for TE and TM modes, respectively.

the resulting coupling factors becomes available. We performed BPM simulations to calculate the coupling factors as explained in [9]. A racetrack resonator system is composed of a racetrack-shaped waveguide optically coupled to an input bus waveguide. The normalized transmitted power from the bus waveguide of the resonator system is given by

$$
I_{t}=\left|\frac{(1-\kappa)^{1 / 2}-\exp \left(-\left(\alpha_{\text {R.T. }} / 2+i \phi\right)\right)}{1-(1-\kappa)^{1 / 2} \exp \left(-\left(\alpha_{\text {R.T. }} / 2+i \phi\right)\right)}\right|^{2} \text {. }
$$

Here, $\kappa$ is the fraction of power coupled between the waveguide and the ring, $\alpha_{\text {R.T. }}$ and $\phi$ are the optical power loss and the phase accumulated per round-trip in the ring, respectively [9]. Using (1), and estimating the total optical loss, including both bending and propagation losses in the resonators, one can arrange the coupling factor to get a desired resonance spectrum.

The fabrication process started by cleaving a $15 \times 15 \mathrm{~mm}$ chip from a whole SOI wafer with top Si layer of $1.00 \mu \mathrm{m}$ and buried oxide layer of $1.00 \mu \mathrm{m}$ on a Si substrate of thickness $625 \mu \mathrm{m}$. Patterns transferred by hard contact photolithography are etched down to $0.5 \mu \mathrm{m}$ on the top Si layer using reactive ion etching [9]. A deposition of 0.9- $\mu \mathrm{m}$-thick $\mathrm{SiO}_{2}$ layer on the chip using plasma-enhanced chemical vapor deposition ended the fabrication.

\section{CharaCterization}

Once the chip was cleaved after a substrate thinning process [9] to a length of $8 \mathrm{~mm}$ and mounted, optical signal from an external cavity tunable laser was coupled to the straight bus waveguides with a 2- $\mu \mathrm{m}$ spot-sized tapered and lensed fiber. The light at the output of the waveguide was collected with a microscope objective and passed through a polarizer of high extinction ratio before reaching a Ge detector. The wavelength of the input light was scanned around $1550 \mathrm{~nm}$ for $4 \mathrm{~nm}$. A number of resonators of bending radii ranging from 20 to $120 \mu \mathrm{m}$ were characterized for both polarizations. Total insertion loss was measured to be

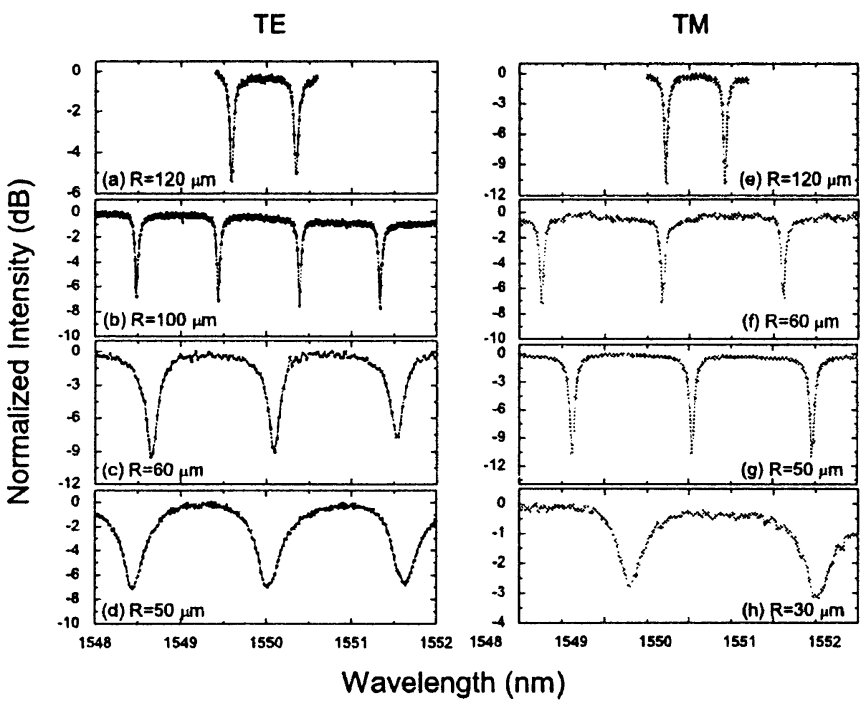

Fig. 2. Measured (a)-(d) TE and (e)-(h) TM transmission spectra of some of the fabricated SOI rib waveguide racetrack resonators.

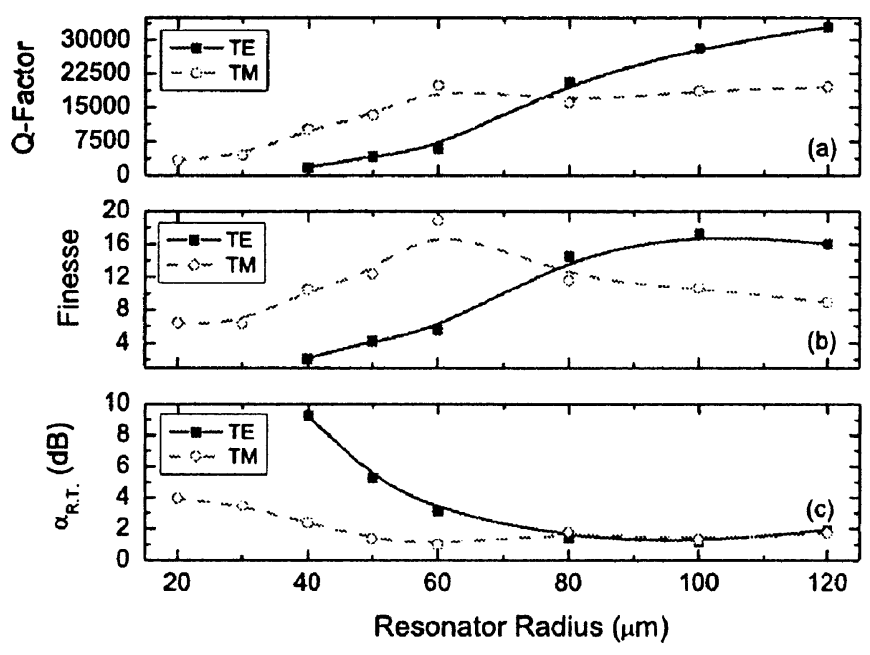

Fig. 3. Measured (a) $Q$-factor and (b) finesse values for the characterized racetrack resonators, and (c) resonator round-trip loss $\left(\alpha_{\text {R.T. }}\right)$ extracted from curve fitting analysis. The lines are to guide the eye.

around $27 \mathrm{~dB}$ with unpolished facets. Resonances for a set of resonators of various radii are displayed in Fig. 2. The FSR of $0.7 \mathrm{~nm}$ at $120-\mu \mathrm{m}$ radius increases to 1.6 and $3.0 \mathrm{~nm}$ for 40 - and $20-\mu \mathrm{m}$ devices, respectively. The waveguide to resonator spacings of 0.7 and $0.8 \mu \mathrm{m}$ were used in the design. The straight coupling sections used to increase coupling from the bus waveguide to the resonator were different for different resonators. It is $36 \mu \mathrm{m}$ for resonators with $60-\mu \mathrm{m}$ radius. TE and TM spectrum of these devices are plotted in Fig. 2(c) and (f).

Quality factors (the ratio of resonance wavelength to bandwidth [11]) of the transmission peaks are plotted as a function of bending radii for both TE and TM modes in Fig. 3(a). The $Q$-factors as high as 20000 for TM at $R \geq 60 \mu \mathrm{m}$ and 28000 for TE at $R \geq 100 \mu \mathrm{m}$ are observed. It is seen in Fig. 3(a), that $Q$-factor decreases dramatically as the bending radius decreases while losses increase. This result can be understood in terms of loss dependence of $Q$-factor. For a resonator coupled to a single 


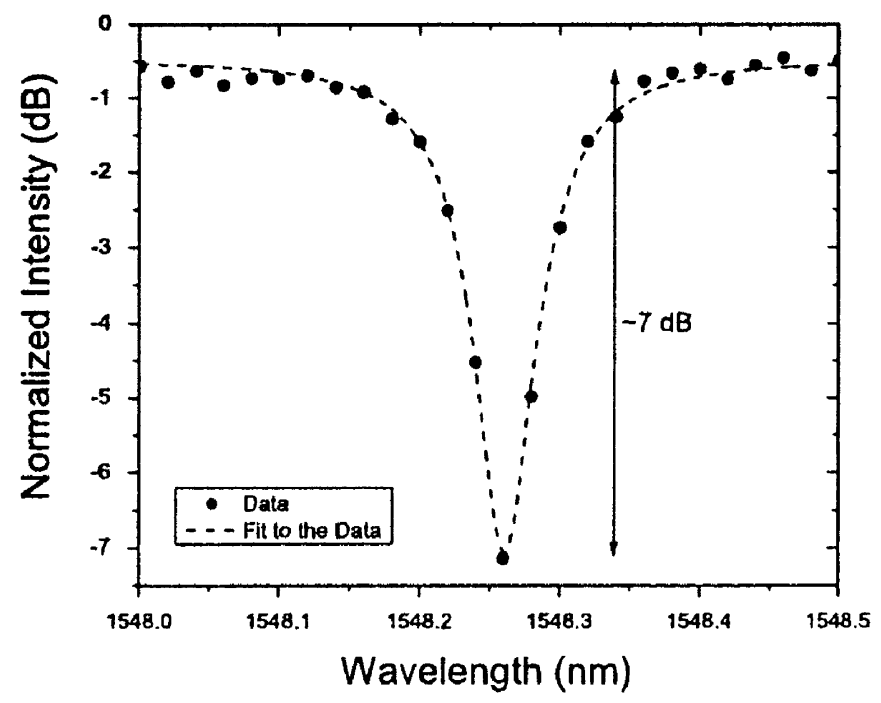

Fig. 4. Fit to the measured TM transmission spectra about $\lambda_{0}=1548.26 \mathrm{~nm}$ using the analytic function in (3) for $R=60 \mu \mathrm{m}$.

bus waveguide, the maximum achievable $Q$-factor can be approximated as $Q_{\max }=\left(2 \pi n_{\text {eff }} / \lambda_{0}\right)(1 / \alpha)$ [4] in terms of effective refractive index $n_{\text {eff }}$ and optical loss coefficient $\alpha . Q_{\max }$ is a strong function of the resonator round-trip attenuation.

We also analyzed the spectra by curve fitting the measured response to the expected response given in (1), to obtain the coupling factor $(\kappa)$ and loss values $\left(\alpha_{\text {R.T. }}\right)$. In the analysis, we used $\kappa$ values calculated using BPM as the initial values. Iterative fitting procedure applied to the data ended in perfect fits. The resulting $\kappa$ values obtained from fit analysis are slightly larger than those calculated using BPM. Consequently, we performed further BPM analysis for gap values which are $0.1 \mu \mathrm{m}$ smaller than the design values. Comparing the calculated $\kappa$ values with those extracted from the experiment suggest that fabrication process results in narrower gaps. A typical example of fitting analysis is shown in Fig. 4. For TM mode at $R=60 \mu \mathrm{m}$, we find that the coupling factor is 0.08 and overall racetrack loss is 0.99 $\mathrm{dB}$ per round-trip after cross-checking with BPM coupling simulations. For the TE polarization, the same parameters are 0.3 for $\kappa$ and $3.14 \mathrm{~dB}$ for loss per round-trip. Higher resonator loss for TE can be understood in terms of high bending loss for this polarization (Fig. 1). Using these results, we calculate $Q_{\max }$ to be about 26700 and 8400 for TM and TE polarizations, respectively. We should note that only half of $Q_{\max }$ is achievable for resonators with high extinction ratios [4]. Larger than achievable $Q$-factors measured imply that these resonators are under coupled, which is supported with observed moderate extinction ratios. The extracted $\alpha_{\text {R.T. }}$. values are plotted as a function of resonator radius in Fig. 3(c). These are total resonator loss per round-trip and include both bending loss due to curvature and accumulated scattering loss. There is an obvious loss increase for resonator of shorter radii which is inferred to be due to very high bending losses. The correlation between extracted $\alpha_{\text {R.T. }}$. in Fig. 3(c) and analytically calculated bending loss in Fig. 1 is obvious. The larger TE bending losses for resonators of smaller radii are as expected in Fig. 1.
Considering that finesse is the ratio of FSR to bandwidth, we show the calculated finesse values in Fig. 3(b). The maximum finesse values of 18.9 and 17.3 are obtained for TM and TE polarizations, respectively. The maximum finesse values are obtained for resonators with smallest $\alpha_{\text {R.T. }}$ as expected. Finally, we note that, as the bending radius increases, radiation loss as well as bandwidth and FSR decrease. However, increasing circumference leads to increase in the accumulated propagation loss, limiting bandwidth and, therefore, the $Q$-factor and reducing finesse as observed for $R=120 \mu \mathrm{m}$.

\section{CONCLUSION}

We have presented design, fabrication, and optical characterization of SOI racetrack resonators with single-mode large cross section waveguides. Using standard optical photolithography technology and single step etch, we achieved bending radii as small as $20 \mu \mathrm{m}$ with FSR as large as $3.0 \mathrm{~nm}$. Particular emphasis was paid to polarization-dependent characteristics of these compact resonators. The different responses of the resonators for TE and TM modes are understood to be due to different boundary conditions experienced by these modes which result in different bending losses and coupling factors. Demonstration of such compact devices opens the possibility of implementing these devices in dense optical circuits.

\section{REFERENCES}

[1] M. K. Chin, C. Youtsey, W. Zhao, T. Pierson, Z. Ren, S. L. Wu, L. Wang, Y. G. Zhao, and S. T. Ho, "GaAs microcavity channel-dropping filter based on a race-track resonator," IEEE Photon. Technol. Lett., vol. 11, no. 12, pp. 1620-1622, Dec. 1999.

[2] D. G. Rabus and M. Hamacher, "MMI-coupled ring resonators in GaInAsP-InP,' IEEE Photon. Technol. Lett., vol. 13, no. 8, pp. 812-814, Aug. 2001.

[3] B. E. Little, J. S. Foresi, G. Steinmeyer, E. R. Thoen, S. T. Chu, H. A. Haus, E. P. Ippen, L. C. Kimerling, and W. Greene, "Ultra-compact $\mathrm{Si}-\mathrm{SiO}_{2}$ microring resonator optical channel dropping filters," IEEE Photon. Technol. Lett., vol. 10, no. 4, pp. 549-551, Apr. 1998.

[4] J. Niehusmann, A. Vörckel, P. H. Bolivar, T. Wahlbrink, W. Henschel, and H. Kurz, "Ultrahigh-quality-factor silicon-on-insulator microring resonator," Opt. Lett., vol. 29, no. 24, pp. 2861-2863, 2004.

[5] T. Baehr-Jones, M. Hochberg, C. Walker, and A. Scherer, "High- $Q$ optical resonators in silicon-on-insulator-based slot waveguides," Appl. Phys. Lett., vol. 86, no. 9, p. 081 101, 2004.

[6] T. Sadagopan, S. J. Choi, S. J. Choi, K. Djordjev, and P. D. Dapkus, "Carrier-induced refractive index changes in InP-based circular microresonators for low-voltage high-speed modulation," IEEE Photon. Technol. Lett., vol. 17, no. 2, pp. 414-416, Feb. 2005.

[7] K. K. Lee, D. R. Lim, L. C. Kimerling, J. Shin, and F. Cerrina, "Fabrication of ultralow-loss $\mathrm{Si} / \mathrm{SiO}_{2}$ waveguides by roughness reduction," Opt. Lett., vol. 26, no. 23, pp. 1888-1890, 2001.

[8] W. R. Headley, G. T. Reed, S. Howe, A. Liu, and M. Paniccia, "Polarization-independent optical racetrack resonators using rib waveguides on silicon-on-insulator," Appl. Phys. Lett., vol. 85, no. 23, pp. 5523-5525, 2004.

[9] I. Kiyat, A. Aydinli, and N. Dagli, "High- $Q$ silicon-on-insulator optical rib waveguide racetrack resonators," Opt. Express, vol. 13, no. 6, pp. 1900-1905, 2005.

[10] V. Subramaniam, G. N. De Brabander, D. H. Naghski, and J. T. Boyd, "Measurement of mode field profiles and bending and transition losses in curved optical channel waveguides," J. Lightw. Technol., vol. 15, no. 6, pp. 990-997, Jun. 1997.

[11] M. K. Chin and S. T. Ho, "Design and modeling of waveguide-coupled single-mode microring resonators," J. Lightw. Technol., vol. 16, no. 8, pp. 1433-1446, Aug. 1998. 